\title{
Dynamic safety assessment of a nonlinear pumped-storage generating system in a transient process
}

\begin{abstract}
This paper focuses on a pumped-storage generating system with a reversible Francis turbine and presents an innovative framework for safety assessment in an attempt to overcome their limitations. Thus the aim is to analyze the dynamic safety process and risk probability of the above nonlinear generating system. This study is carried out based on an existing pumped-storage power station. In this paper we show the dynamic safety evaluation process and risk probability of the nonlinear generating system using Fisher discriminant method. A comparison analysis for the safety assessment is performed between two different closing laws, namely the separate mode only to include a guide vane and the linkage mode that includes a guide vane and a ball valve. We find that the most unfavorable condition of the generating system occurs in the final stage of the load rejection transient process. It is also demonstrated that there is no risk to the generating system with the linkage mode but the risk probability of the separate mode is 6 percent. The results obtained are in good agreement with the actual operation of hydropower stations. The developed framework may not only be adopted for the applications of the pumped-storage generating system with a reversible Francis turbine but serves as the basis for the safety assessment of various engineering applications.
\end{abstract}

Keyword: Pumped-storage generating system; Francis turbine; Dynamic safety analysis; Risk probability 
